\title{
Transient measurement results of pulse propagation in large GTEM cells
}

\author{
H. Thye ${ }^{1}$, G. Armbrecht ${ }^{2}$, and M. Koch ${ }^{1}$ \\ ${ }^{1}$ Institut für Grundlagen der Elektrotechnik, Leibniz Universität Hannover, Germany \\ ${ }^{2}$ Institut für Hochfrequenztechnik und Funksysteme, Leibniz Universität Hannover, Germany
}

\begin{abstract}
This contribution deals with the results of a transient measurement campaign incorporating ultra-wideband (UWB) pulses applied to a large GTEM cell. The main purpose is to analyse the distortion effects on such a feeding pulse when transformed into a field pulse inside the cells testing volume. We will investigate if the TEM field distribution is interfered by multimode propagation, that may lead to location-dependent pulse distortion and ringing. Finally, conclusions on the applicability of GTEM cells for standardized transient EMC measurements will be drawn.
\end{abstract}

\section{Introduction}

In recent years there has been an increasing demand for immunity testing with fast transient signals, especially regarding UWB signals (rise time about $400 \mathrm{ps}$ ). This particular interest is driven by the increasing application of UWB signals both in military and in civilian applications. The double exponential pulse (DEP) represents a prominent UWB pulse shape for EMC immunity considerations. Because of the short rise times the pulses have a instantaneous frequency spectrum up to the Gigahertz range.

The abbreviation GTEM cell stands for Gigahertz Transversal ElectroMagnetic cell and implies that the upper frequency limit up to which the TEM mode is dominant extends to the $\mathrm{GHz}$ range., so it falls into consideration as a measurement instrument for these signals. However, it is well known, that the GTEM as well as the Crawford cell loses its pure TEM behavior above certain frequencies (Wilson, 1995). Extensive investigations in the frequency domain have shown that these phenomena are caused by the excitation of higher order modes (Koch, 1998). In this article the analysis focus on the consequences of the expected excita-

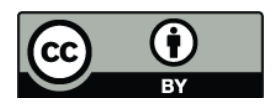

Correspondence to: $\mathrm{H}$. Thye (thye@ieee.org)
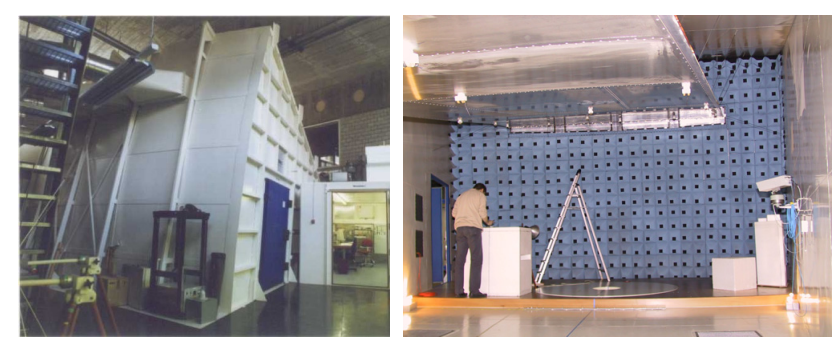

Fig. 1. Outer view and inner view of the GTEM 3750.

tion of higher order modes. Depending on the distinct observation point in the testing volume the measurement results of the feeding pulse are considered by means of pulse distortion and ringing effects. The investigated GTEM 3750 operated by armasuisse, located in Bern (Switzerland). The total length is $15.51 \mathrm{~m}$ and the height of the septum in the testing volume is $3.22 \mathrm{~m}$. Figure 1 illustrates the large dimensions.

The article is structured as follows: In Sect. 2 the used measurement environment setup will be introduced with all hardware components. After the presentation of the defined coordinate systems and the observation points in the cell, the section ends with an introduction of the used excitation signal. After that, Sect. 3 will show selected measurement results. On the basis of these results first general conclusions can be made.

\section{Measurement setup}

Figure 2 shows the complete drafted measurement setup with all components like probes, cables, sampling oscilloscope. Inside the cell, E- or H-field sensors are fixed on an electromagnetic non-visible EMC-tripod. The connection to the receiver is provided through optical waveguides in order to minimize the additional field distortion as by metallic waveguides. The optical receiver transforms the received signals into voltages, which can be displayed in the sampling

Published by Copernicus Publications on behalf of the URSI Landesausschuss in der Bundesrepublik Deutschland e.V. 


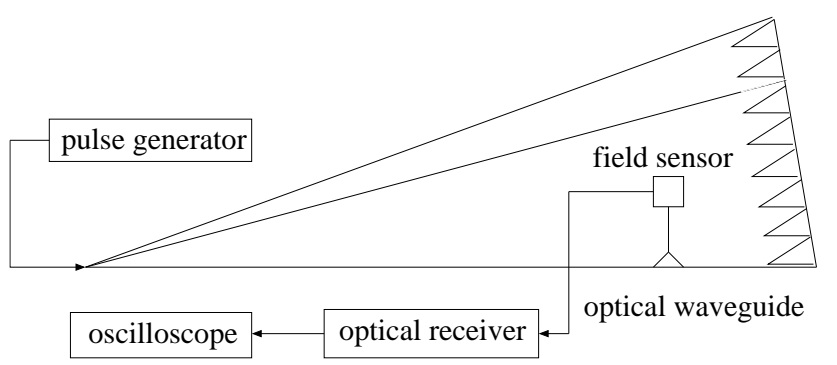

Fig. 2. View of the measurement setup.
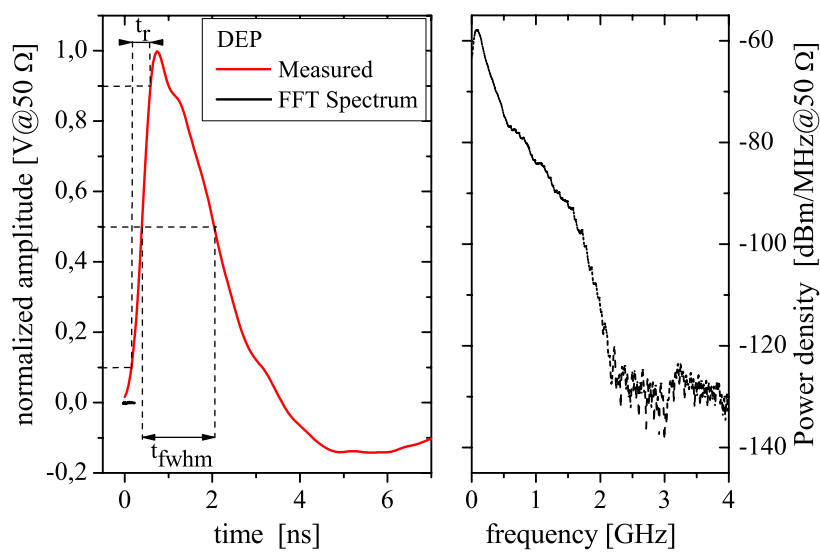

Fig. 3. Applied DEP function in time and frequency domain.

oscilloscope with a maximal resolution of $20 \mathrm{GS} / \mathrm{s}$. The utilized signal source is the commercial pulse generator HYPS by Grant Applied Physics. It sends a double exponential pulse (DEP) with a turnable peak value up to nearly $1.5 \mathrm{kV}$, which is a common pulse form for transient EMC testing procedures. This voltage leads to a maximum electric field strength of approximately $400 \mathrm{~V} / \mathrm{m}$ in the testing volume of the cell. Figure 3 shows the normalized amplitude in time domain and the spectral power density of the output signal of the introduces signal generator into a $50 \Omega$ match. To measure the signal, attenuators with $60 \mathrm{~dB}$ attenuation were placed between the generator and the oscilloscope. The DEP has a rise time $t_{r}$ of about $400 \mathrm{ps}$ and a full width half max value $\left(t_{f w h m}\right)$ of $1.7 \mathrm{~ns}$. Due to this values the DEP has a limit frequency to noise at about $2.5 \mathrm{GHz}$. Furthermore the pulse parameters rise time $\left(t_{r}\right)$ and full width half max value $\left(t_{f w h m}\right)$ are each identified.

Figure 4 shows the defined coordinate systems. Due to the practical consideration of the measurement setup the $\mathrm{z}$ axis of the dashed coordinate system $\left(x^{\prime}, y^{\prime}, z^{\prime}\right)$ is defined parallel to the ground of the cell because the field sensor is originally determined in the dashed coordinate system. In the main coordinate system $(\mathrm{x}, \mathrm{y}, \mathrm{z})$ the $\mathrm{z}$-axis is defined in the geometrical center of the cell. Because of that the xy-cross section, at any z-coordinate, is proportionally scaled, which

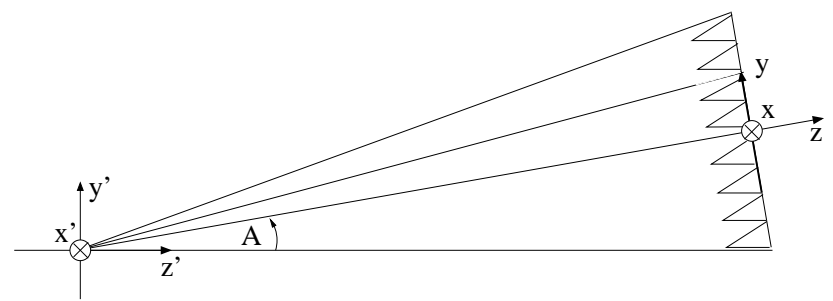

Fig. 4. Definition of the used coordinate systems.

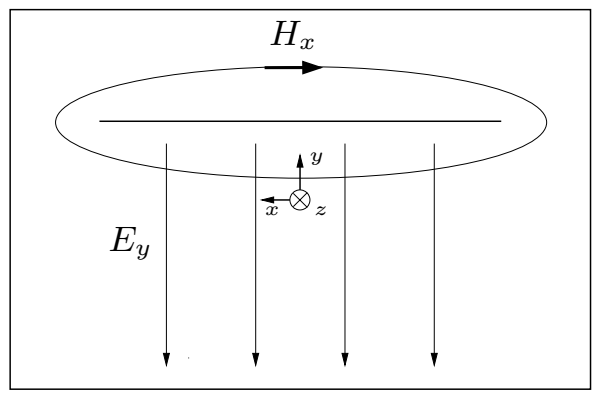

Fig. 5. Main electromagnetic components in the cross section of the cell.

leads to the typical pyramidal shape of a GTEM cell. In the following section all field quantities are specified with this system.

\section{Results}

In this section the measurement results will be discussed. First, the measurement results are extracted at distinct probe positions in the testing volume of the cell. After that, contour plots show the field distribution at one complete cross section. Provided that the TEM mode is dominating the magnetic x-component $\left(H_{x}\right)$ and the electric y-component $\left(E_{y}\right)$, according to the introduced coordinate system, these are the main field components. As depicted in Fig. 5, $H_{x}$ encloses the septum of the cell, whereas $E_{y}$ is straight line orientated from the septum to the cells floor. In this paper, exemplarily $H_{x}$ and $H_{y}$ will be considered for presentation.

As already introduces by Koch (1998) the field is extracted at defined field points (P1-P10) in the testing volume of the cell. The positions are located in two different xy-planes, varying the first cross-section $\left(y_{1}^{\prime}=0.88 \mathrm{~m}\right.$ and $\left.z_{1}^{\prime}=8.51 \mathrm{~m}, \mathrm{P} 7-\mathrm{P} 10\right)$ to the second cross-section $\left(y_{2}^{\prime}=1.25 \mathrm{~m}\right.$ and $\left.z_{2}^{\prime}=12.11 \mathrm{~m}, \mathrm{P} 7-\mathrm{P} 10\right)$. The points are located in the center of the cell in $\mathrm{x}$-direction with an incremental distance $\Delta x=0.5 \mathrm{~m}$.

Figure 7 shows the first results of the normalized magnetic field component $H_{x}$ at the points $\mathrm{P} 1$ and P7, which only differ in the z-coordinate. As predicted the amplitudes of the pulses declines with increasing distance from the feeding 


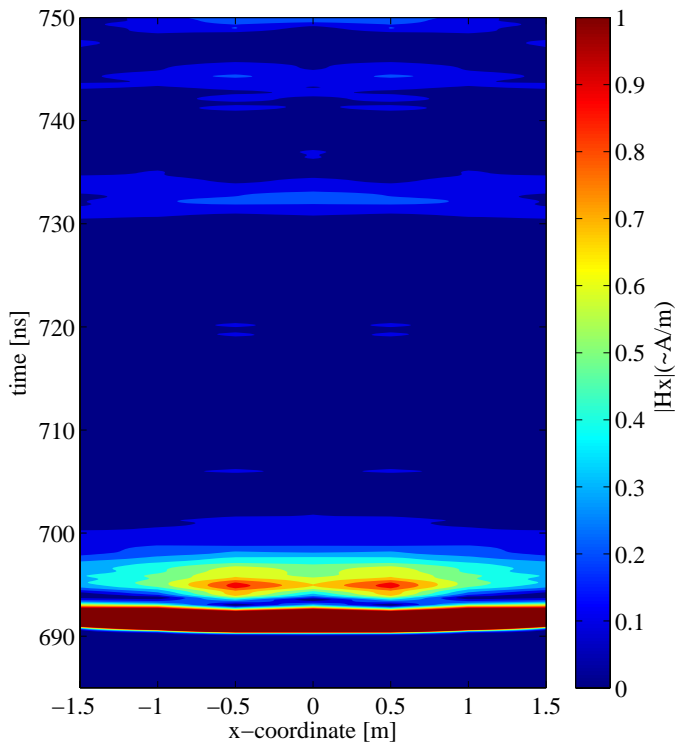

Cross-section 1 at $\mathrm{z}^{\prime}=8.51 \mathrm{~m}$

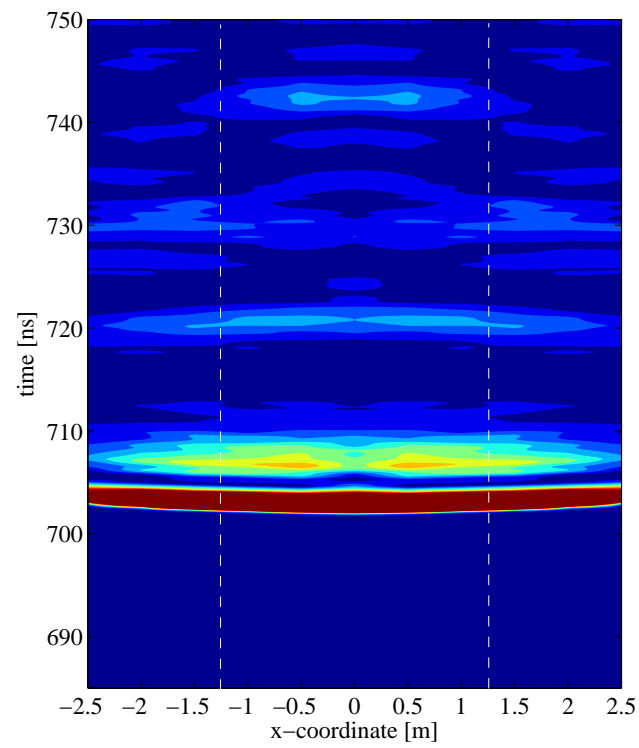

Cross-section 2 at $\mathrm{z}^{\prime}=12.22 \mathrm{~m}$

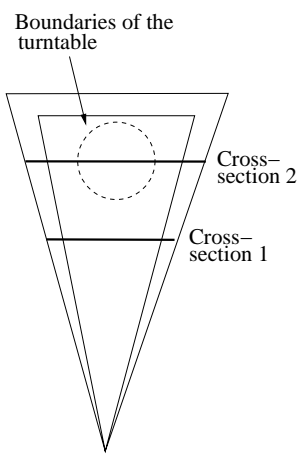

Draft of the GTEM cell

Fig. 6. Magnitude of the normalized measured time dependent magnetic x-component in two cross sections of the GTEM cell.

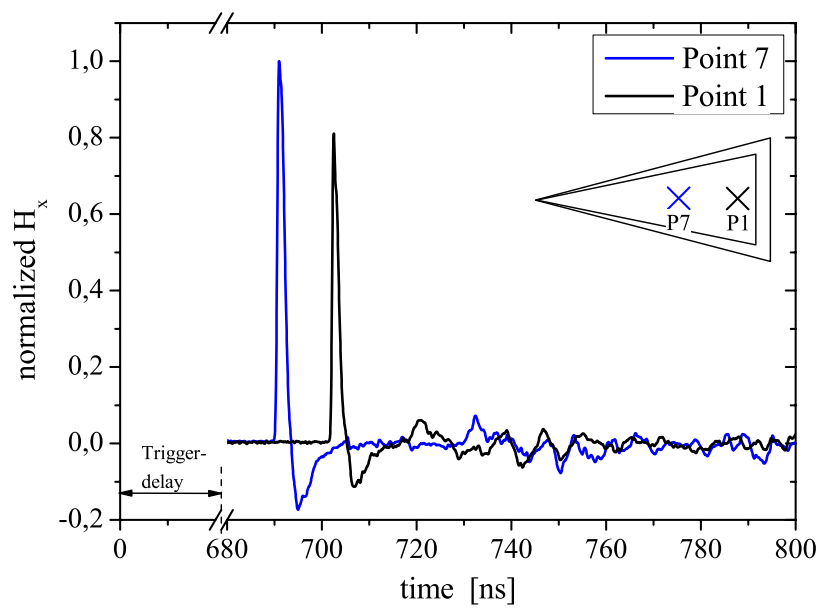

Fig. 7. Measured $H_{x}$ in two points with absolute time scaling (DEP Excitation).

port. Both results are equally retarded by a trigger delay of about nearly $680 \mathrm{~ns}$. Due to the constant trigger delay, that similarly effects empty results, it is possible to measure the time dependent reflections with absolute values.

As mentioned in the introduction, a major interest of the investigation is the distortion of the excited voltage pulse in the testing volume of the cell. Therefore, the normalized exciting voltage pulse and the normalized measured $H_{x}$ at point $\mathrm{P} 1$ are compared. As shown in Fig. 8 there is a sound correlation of these signals. The rise times and pulse widths of both

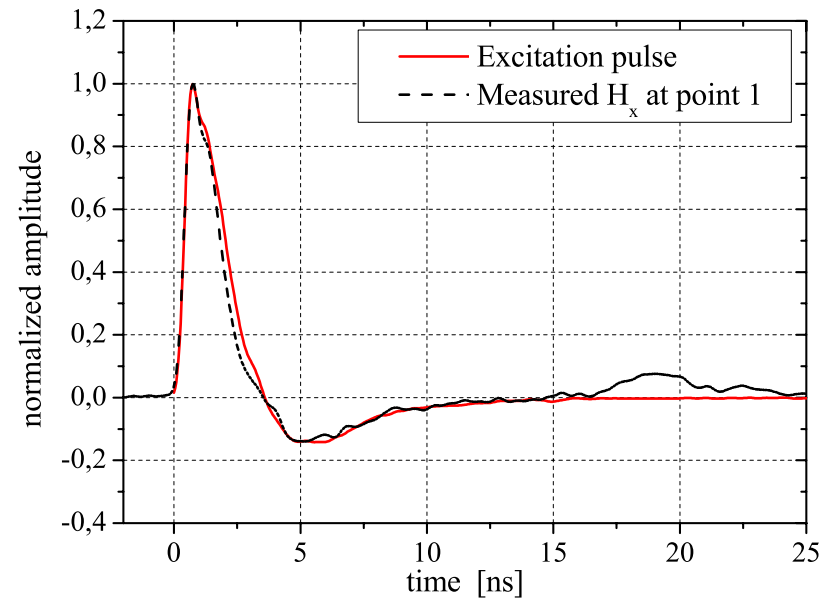

Fig. 8. Comparison of normalized incoming pulse and measured $H_{x}$ at point 1 (DEP Excitation).

impulses are nearly identical. Only in the area of the falling edge it is noticeable that the measured field pulse is narrower than the excitation pulse. An explanation is the expansion of the excitation pulse through the attenuators which are necessary for its measurement (see Sect. 2). Significant differences arise primarily as reflections arrive (ringing effect). The measurement setup includes the field sensors, which exhibit an unknown transfer function. If the field pulse had been broader that the excitation pulse, a field sensor could not have reversed this propagation. 


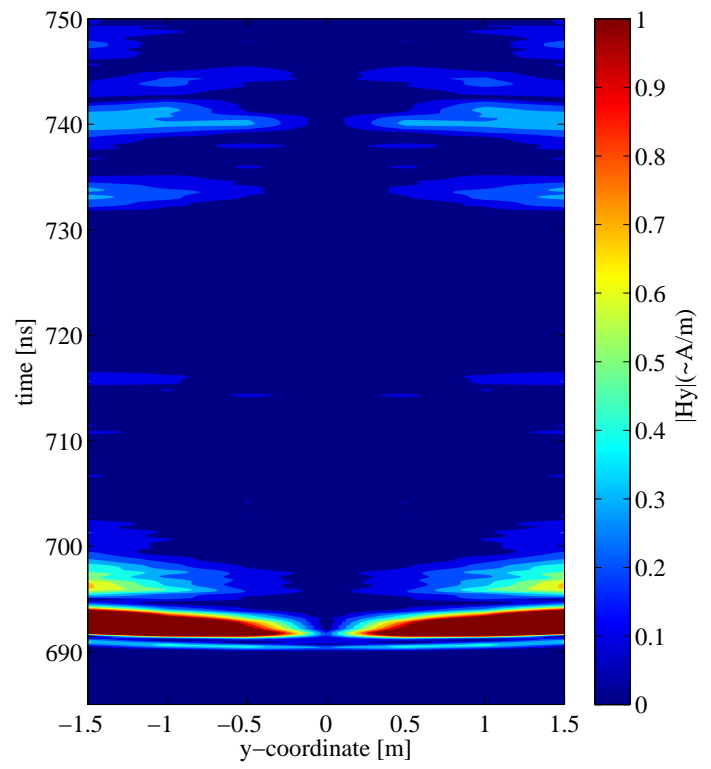

Cross-section 1 at $\mathrm{z}^{\prime}=8.51 \mathrm{~m}$

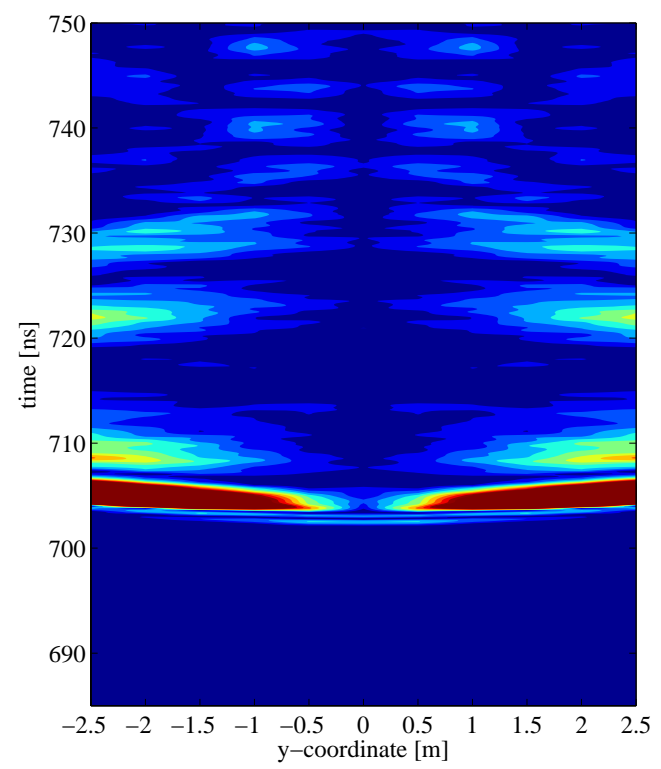

Cross-section 2 at $\mathrm{z}^{\prime}=12.22 \mathrm{~m}$

Fig. 9. Magnitude of the normalized measured time dependent magnetic y-component in two cross sections of the GTEM cell.

In order to get a better impression of the local dispersion of the electromagnetic field in the cell, the field allocation in two defined cross sections are investigated. Hence, Fig. 6 shows a contour plot of the two cross sections, which includes the shown field points P1 and P7. The normalized amplitude of $H_{x}$ is presented time dependent in Fig. 6a (cross section 1, P7-P10) and Fig. 6b (cross section 2, P1-P6). Due to the symmetry properties of GTEM cells, the results to the yz-level can be mirrored. With these contour figures, the displacement in the time domain at the outer regions in the cell and the relationship of the amplitudes (included the reflections) can be analyzed at once. For practical considerations the DUT is placed on the turntable in the testing volume during the measurements. Figure 6 signifies the dimensions of this turntable with dashed lines. Additionally, Fig. 9a and b shows the magnetic y-component $\left(H_{y}\right)$ in the same cross sections (1 and 2). Because of the lower absolute value of $H_{y}$ in relation to $H_{x}$ (about $3.8 \%$ of the peak value) the amplitude is independently normalized to make the pulse propagation visible.

\section{Conclusions}

In this paper pulse propagation measurements directly in time domain were conducted in a large GTEM cell. Selected electromagnetic field distributions in the testing volume of reflections the cell were presented in one- and twodimensional plots. It was shown that the primary pulse in the GTEM cell displays a nearly distortionfree transformation of the voltage pulse. After the primary pulse passes through the cell, a number of reflections with apparent attenuated amplitudes are visible. This point and the fact, that the main field components $\left(H_{x}\right.$ and $\left.E_{y}\right)$ are dominating in relation to the other, leads to the conclusion, that the TEM mode dominates by transient measurements in GTEM cells. Further investigations are necessary to verify this statement.

Acknowledgements. The authors would like to thank the armasuisse, especially H. Schär and A. Horvarth for the supply of the measurement setup and the support during the measurements.

\section{References}

Camp, M., Garbe, H., and Nitsch, D.: Influence of the technology on the destruction effects of semiconductors by impact of EMP and UWB pulses, IEEE International Symposium on EMC 2002, pp. 87-92, USA, Minneapolis, 2002.

Koch, M.: Analytische Feldberechnung in TEM-Zellen, Dissertation Uni Hannover, 1998.

Wilson, P.: On Correlating TEM-cell and OATS Emission Measurements, IEEE Transactions on Electromagnetic Compatibility, 1995. 